\title{
ANTROPOLOGIA DE LA POBREZA. LOS GITANOS Y EL CASO DEL ASENTAMIENTO SEVILLANO DE "LOS BERMEJALES"
}

Ramón REIG*

\section{INTRODUCCION}

El pueblo o raza gitana es uno de los exponentes más claros de la antropología de la pobreza que se da en las grandes ciudades o sociedades complejas. A estos grandes núcleos de población, los gitanos acuden en masa con la esperanza de vivir a costa de las "migajas" de la amplia clase media urbana. En la ciudad, el gitano vive de subtrabajos o, mejor dicho, de ocupaciones, como la venta de flores, frutos del campo, recolección y venta de cartones y papel, venta de objetos en sitios estratégicos (como los conocidos casos de los paquetes de pañuelos de papel en los semáforos, que también es tarea de payos), o, simplemente, subsisten por obra y gracia de la caridad. (Puede constatarse ahora una especie de legalización de la caridad con el llamado salario social, con el que, al igual que en la Edad Media, y salvando distancias, queda "constituido" el estamento pobre).

Todos los elementos de una familia trabajan de alguna u otra forma en éstas y en otras ocupaciones. No hace falta decir que la formación cultural gitana es bajísima y que son objeto de una especie de "repulsión" psicológica y real por parte de los demás ciudadanos. Conocido es de sobra el caso de los gitanos de Martos (Jaén). En esta localidad existe o existía un asentamiento gitano que fue asaltado literalmente por vecinos de la localidad que llegaron a incendiar las modestísimas viviendas gitanas. En otros lugares de Andalucía numerosos padres de alumnos se niegan a que en el colegio de sus hijos estén presentes gitanos niños. Y en Sevilla, además de darse también este caso, encontramos un rechazo vecinal a los gitanos cuando el Ayuntamiento planea acometer un plan de erradicación del chabolismo para llevar a sus habitantes - gitanos por lo general- a otros barrios, que suelen ser barrios de la denominada clase trabajadora.

\footnotetext{
* Profesor de la Facultad de Ciencias de la Información. Universidad de Sevilla.
} 
Al margen del rechazo puramente étnico -discutible a mi juicio-tenemos un repulsa no a lo que es sino a aquello que el gitano representa para el payo, fruto : su vez de una trayectoria histórica concreta.

Aunque sea de forma sucinta, vamos a intentar a través de este trabajo intro. ducirnos en el mundo gitano, mediante una tarea de campo llevada a cabo en e asentamiento sevillano de "Los Bermejales". En dicho asentamiento, el Ayuntamiento sevillano ha tratado de introducir elementos humanos profesionales expertos en animación sociocultural. Sin embargo, hemos hablado personalment $\epsilon$ con algunos de ellos y coinciden en la falta de resultados. No es de extrañar e] hecho ya que una comunidad que, en primer lugar, tiene imperiosas necesidades de subsistir alimenticiamente, laboralmente, no es permeable en exceso a este tipo de actividades. Más bien creemos que la acción municipal obedece a un intento de "lavarse la conciencia" ante un gravísimo problema que no se quiere c no se puede solucionar en profundidad.

Mostraremos pues a lo largo de estas líneas lo que hemos recogido en visitas al asentamiento de "Los Bermejales". No obstante, parece oportuno, para que el trabajo tenga un matiz más concreto y completo, esbozar primeramente unos datos sobre la historia gitana en España, pasar en segundo lugar a tratar sobre las características ecológicas o de entorno, podríamos decir, del asentamiento, y terminar con las impresiones y opiniones recogidas en el lugar.

Por último, un capítulo final nos permitirá acercarnos más, siquiera sea desde la estadística, a la realidad del pueblo gitano en su entorno geográfico de Andalucía, según datos de las Administraciones Autonóma y Central.

Quisiéramos añadir que el acontecimiento Expo'92 conlleva la desaparición del asentamiento gitano de "Los Bermejales" para construir en la zona, entre otros elementos arquitectónicos, un hotel de alto "standing". No obstante, cuando escribimos estas líneas -inicios del mes de junio de 1990- aún no ha desaparecido, al contrario de lo que sucedió en 1989 con otro asentamiento gitano: el de Chapina, situado cerca de la sede de la Expo (Isla de La Cartuja). Sus habitantes recibieron algo más de un millón de pesetas por familia y fueron abandonados a su suerte. Las nuevas obras de encauzamiento del río obligaron a desmantelar un núcleo de población que subsistía sobre todo de la venta de chatarra y la mendicidad.

\section{GITANOS: DATOS PARA UNA HISTORIA}

Dos importantes nombres de la cultura andaluza, Félix Grande y Juan Peña "El Lebrijano", llevaron a la escena hace una docena de años una obra, que 
también "se sacó" en disco, y que lleva por título "Persecución”. Era un título significativo de la historia del pueblo gitano. En 1499 y en Medina del Campo, los Reyes Cáólicos, tan ortodoxos de la pureza religiosa, dictaron la famosa Pragmática en el Castillo de la Mota. La Pragmática representó el inicio de una serie de leyes contra el pueblo gitano, el inicio de una persecución que, de una manera mucho más tamizada e invisible, aunque a veces, como hemos dicbo en la "Introducción", es bien visible, llega hasta nuestros días.

Según algunos indicios, el pueblo gitano llegó al territorio ibérico peninsular corriendo el año 1425, portando una cultura y unas costumbres milenarias que chocaron claramente con las rígidas estructuras establecidas. Hay, sin embargo, otras fuentes que indican o que consideran a estos gitanos llegados en el siglo $\mathrm{XV}$ como una especie de segunda remesa, ya que antes habían entrado procedentes de Africa (los citados anteriormente lo habían hecho a través de Francia quizá originarios de la India) otros grupos que son conocidos a través de las fuentes como "gitanos".

Dentro de la escasez de datos fiables, suele decirse que el pueblo gitano procede de la India de donde hubo de salir presionado principalmente por el empuje turco. Actualmente, en la India, sobre todo en la localidad de Orissa, pueden verse grupos de familias nómadas que viajan y viven en carros cubiertos por velas y se consideran descendientes de los gitanos originarios. La raíz india de procedencia se hunde incluso en aspectos legendarios de la literatura.

En el siglo XV español, nos dice Benigno González ', "los gitanos se sentían a gusto y en todas partes se dejaban ver menos en el penitente Camino de Santiago, como si trataran de levantar el mapa de los caminos y encrucijadas, de trochas y veredas. Mientras la religión los protegió perpetuando para ellos la tregua de Dios, la vida no les fue mal del todo. Pero su amor a la independencia, su afición a lo maravilloso, su tendencia a anteponer lo ideal a lo real, su desprecio al rendimiento del trabajo penoso, pero lucrativo, prefiriendo la aventura o la misma pobreza y alguna cosilla más que no hace al caso -y si lo hace es mejor no meneallo-, acabó por mosquear a los nativos. Duros tiempos empezaron para los gitanos".

A nuestro juicio, Benigno González se precipita en sus afirmaciones. Los tiempos duros empezaron para los gitanos en el momento de pisar tierra española. Se trataba de una minoría que no estaba encuadrada en la jerarquía social. Los gitanos no odiaban el trabajo, igual que no lo odiaban muchos payos que en aquella época -como en la actual-carecían de posibilidades de trabajar. Aparte

1. Benigno González: "Las peregrinaciones gitanas en el Siglo XV". Diario ABC, Sevilla, 1978. Diciembre. 
la idiosincrasia del propio pueblo gitano, lo que sucedió es de sobra conocide Sus profesiones eran liberales y no encontraron lugar en un mundo clarament estratificado, dominado por los gremios y por los señoríos.

Por otra parte, las asimilaciones que los poderes oficiales intentaron, dentrı siempre de la ortodoxia católica, pretendían desprender a este pueblo de su propias características consuetudinarias. Y este proceder aún se sigue haciend، en la actualidad. Detengámonos en un ejemplo.

Durante los días 8, 9 y 10 de septiembre de 1978, se celebró en Sevilla lo qu se denominó pomposamente "Peregrinación Universal de Gitanos a Sevilla". Et este acontecimiento intervino de una manera considerable el Opus Dei, desig nándose como objetivo de la peregrinación "postrarse a los pies de la Macare na".

Con el Opus Dei al fondo, la llamada Dirección Nacional de Apostolads Gitano, editó un folleto ilustrativo del acontecimiento, que repartió entre lo: protagonistas. En él podía leerse: "Una peregrinación es, ante todo, una march: colectiva y deberá darse prioridad a lo colectivo. Pero esto no excluye la promo ción individual, en particular la preparación a los sacramentos. Cuidando de qut no se haga una preparación demasiado rápida se podrá encontrar en las peregri. naciones la ocasión de llevar a ciertos gitanos a la Primera Comunión o lá Comunión Pascual, a la Confirmación, al matrimonio religioso"?

En el siglo XV y posteriores estas pretensiones no se hacían de una forma tar larvada. La evolución de los tiempos aligera las formas de dominación y esté Dirección Nacional de Apostolado Gitano flexibiliza sus deseos, aunque sigue hablando de "matrimonio religioso", como si las demás formas de matrimonic que existen, y que los gitanos llevan a la práctica, carecieran de ese matiz.

Con motivo de la peregrinación universal a Sevilla a la que venimos refirién. donos, uno de los "líderes" gitanos, el llamado Tío Peret, declaró a la prensa er aquel momento ${ }^{3}$ que el gitano era ciertamente religioso, pero no por ello debía ser católico. A pesar de todo, y en teoría, a Sevilla se vino hace doce años a adorar a la Macarena y, aunque existen numerosos gitanos que se han contagiadc de la devoción hacia la universal imagen, los resultados finales de la peregrinación pudieron palparse: de un total de treinta mil peregrinos que se esperaban llegaron tres mil escasos ${ }^{4}$.

Las fuentes más sólidas, que denotan la presencia gitana en la Península a partir del siglo XV, refieren también la sorpresa que causaban aquellas gentes

2. Véase: Reig, Ramón: "Gitanos, la etema peregrinación". Revista "Algarabía" n." 0. Málaga, noviembre, 1978.

3. Diario "Suroeste". Sevilla, 6-9-1978.

4. Reig, Ramón: art, cit. 
"extrañas y vagabundas" que suscitaron al mismo tiempo curiosidad y rechazo. La literatura de la época empezó a hacerse eco de la novedad y Gil Vicente, Lope de Rueda y Cervantes, entre otros, se ocuparon de los nuevos habitantes tratándoles en ocasiones con simpatía hacia ese estilo bobemio de vida que contrastaba con una España de gorguera y capa negra.

A pesar de los decretos represivos los resultados no fueron apenas positivos para sus emisores. Los gitanos continuaron dedicándose al comercio de caballerías y otros pequeños oficios como los de esquilador y herrador. Las mujeres por su parte ejercían la mendicidad y leían la buenaventura.

Domínguez Ortiz dice ${ }^{5}$ que la falta de unos ingresos regulares los impulsó con frecuencia el merodeo y al bandidaje agrupándose en partidas numerosas. Las Cortes de Castilla protestaron numerosas veces y se dictaron leyes en 1575 y 1639 por las que se condenaba a galeras a todos los gitanos varones. Sin embargo, había pruebas inequívocas de que el gitano era asimilable: en Sevilla, Granada y otras ciudades vivían gitanos avecindadosz y ejerciendo oficios. Según el historiador citado, a los gitanos procedentes de Asia, que además habrían pasado por Egipto tomando el nombre de egipcianos, de donde se derivaría la denominación actual, se les añadieron elementos marginados como esclavos fugitivos, moriscos deseosos de evitar la orden de expulsión, aventureros, pícaros, inadaptados y todo un mundo fuera de la ley.

En aquella época, a los peninsulares extrañó la total indiferencia religiosa de este pueblo que prácticamente, y a diferencia de judíos y moriscos, no hacía gala de ninguna religión. Afirmaban ser cristianos y de esta manera se libraban del brazo represivo de la Inquisición que se ocupaba tan sólo de las creencias y no de la práctica. Era opinión general que la mayoría ni se bautizaba ni cumplía los preceptos de la Iglesia aunque no se pudiera probar por carecer normalmente de domicilio.

Algunos prelados establecieron sobre ellos cierta vigilancia, como hizo el de Almería, ordenando en 1655 que "les pidan la razón de donde se casaron y quién bautizó a sus hijos. Prohíbanles hablar su lenguaje, traer su traje, bailar y cantar la buenaventura y andar en compañías". Otro prelado, el de Málaga, ordenaba por las mismas fechas que los curas averiguasen si estaban bautizados, casados y cumplían con la Iglesia.

No se hizo en realidad ningún esfuerzo serio para resolver la cuestión gitana que se le entregó al siglo XVIII con caracteres parecidos a los de anteriores épocas. Así sucedieron episodios como el de 1746 en que el primer Borbón,

5. Antonio Domínguez Ortiz: "El antiguo régimen: los Reyes Católicos y los Austrias". Historia de España, Vol. III. Alianza-Universidad, Madrid, 1977, pp. 181-193. 
Felipe $V$, publica una ley que permite la persecución gitana hasta dentro de las iglesias, ley que no fue derogada hasta principios del siglo XX.

De todas formas, la marginación es patente que aún continúa. En la peregrinación o intento de peregrinación universal a Sevilla a que antes nos referíamos. el pueblo gitano volvió a ser maltratado en el sentido de que sus ubicaciones físicas en Sevilla volvieron a estar en zonas marginales y con carencias infraestructurales.

A lo largo de estas líneas creo que han podido constatarse unas señas de etnicidad gitana. No sólo poseen una forma de vivir sino una lengua, el caló, y una forma cultural: el flamenco o el cante propiamente gitano. "El flamencc - dice Félix Grande - no ha nacido al calor de los Estados ni de las culturas dominantes. Ha brotado de un pueblo despreciado, que a veces es gitano, a veces andaluz de abajo, y siempre en una geografía ante la que, desde hace cinco siglos, los Estados no se desviven. En los sucesivos repartos de hambre y prorrateos de olvido, Andalucía viene invariablemente sufriendo la mejor tajada. Así ha venido siendo, así continúa siendo. Tanto olvido llega a parecerse al desprecio".

Estas palabras de Félix Grande fueron escritas hace algunos años. Todavía muchos las suscriben. Pero es evidente que, al menos a niveles de datos macroeconómicos, la situación ha cambiado tanto desde el punto de vista español como europeo (entrada de España en la CEE con sus repercusiones en Andalucía en cuanto a aportación de los Fondos Estructurales). Sin embargo, lo cierto es que, a simple vista, el estado de cosas en Andalucía sigue siendo delicado. Y si nos referimos al mundo gitano observamos que poco o muy poco ha cambiado el paisaje.

Algunos autores, como el flamencólogo José Luis Ortiz Nuevo, han llegado a afirmar ${ }^{7}$ que el pueblo gitano es el símbolo del pueblo andaluz. Las reacciones a todo un estado de cosas se concretaron en cantos interpretados en solitario sin acompañamiento de guitarras, construidos sobre viejas formas de romances, repletos de angustias, persecuciones - como las que ha cantado Juan Peña "El Lebrijano" en el disco y la obra teatral a que hacíamos alusión al comienzo de este apartado-, y crueldades. A estos gritos se les denominó Tonás.

Unas tonás cuyas letras bien podrían reflejar el medio millón de gitanos que murieron a manos de los nazis y que muy pocos libros de texto destacan. Todos conocemos las persecuciones de que fueron objeto los judíos pero sabemos en menor medida la cuestión gitana en la Segunda Gran Guerra. Hoy día hay

6. Félix Grande: "Para un dossier sobre la pena del gitano: Lágrimas testarudas". Tiempo de Historia, Año IV, núm. 45, agosto 1978, pp. 83-84.

7. Manifestaciones orales a quien suscribe. 
esparcido por Europa un número diez u once veces superior al de las víctimas nazis. En la Guerra Civil española se sucedieron combates y héroes en ambos lados de la contienda. En la Segunda Guerra Mundial no hubo héroes gitanos porque todos murieron indefensos. Ante los nazis y proporcionalmente, cayeron el doble de individuos gitanos que españoles cayeron en ambos bandos durante la Guerra Civil en $1936^{*}$.

Antes de finalizar este capítulo de aproximación histórica nos gustaría recoger brevemente las teorías de un notable especialista en la materia: el profesor Bernard Leblon, de la Universidad de Perpignan. El día 22 de mayo de 1990 Leblon pronunció en la Facultad de Geografía e Historia de la Universidad de Sevilla una conferencia que, bajo el título de "Historia de los gitanos en España y orígenes del flamenco", organizó el Aula de Flamenco de la Universidad Hispalense, coordinada por el profesor Miguel Ropero.

En dicha conferencia, a la que asistimos, el profesor Leblon se refirió en primer lugar a las raíces del flamenco, mostrando sus dudas sobre la rigurosidad de las teorías que hablan de buscar dichas raíces en la música religiosa bizantina (una civilización de fugaz paso por Andalucía) e inclinándose más por el canto religioso hispano (conocido también por mozárabe en denominación a su juicio apresurada por cuanto este canto se daba antes de la llegada del Islam), si bien sus convicciones más profundas se dirigían hacia la música oriental, andalusí y húngara.

Lo cierto es que, siempre según Bernad Leblon, en Europa Occidental los gitanos se presentan como músicos (tocadores de laúd en Hungría en la segunda mitad del siglo XV, por ejemplo), procedentes de la India y llegados a través de Grecia y Turquía. El origen de esta peregrinación entra ya en el terreno de la leyenda y parece llegar hasta el siglo $\mathrm{V}$.

En España participan musicalmente en fiestas populares, especialmente en el Corpus. A finales del XVI, ocho gitanas, acompañadas por dos o seis bailarines gitanos y músicos, participan habitualmente en el Corpus, sobre todo en Granada. En esta ciudad, se daban además otros festejos anuales que parecían "lamentar" la llegada de los Reyes Católicos en 1492. Aunque dichos festejos son prohibidos a finales del XVI los moriscos van a continuar de alguna manera realizándolos, lo que añadirá un factor más en favor de la expulsión.

La política institucional española respecto a los gitanos va a ser distinta o, al menos, va a contar con peculiaridades respecto a otras naciones europeas. Mientras que en otras naciones no parece importar tanto su nomadismo, en España las medidas de los Reyes Católicos exigen su asentamiento o su marcha del país.

8. Art. cit. de Félix Grande, pp. 85-90. 
Así, bajo el espíritu de esta política, mientras que los moriscos son expulsa dos, a los gitanos se les va a hacer la existencia muy difícil (una especie di expulsión indirecta, no decretada tan drásticamente como en el caso morisco) Paulatinamente se van creando residencias especiales para gitanos, sobre todo et el siglo XVIII. En esta centuria incluso se pena con la muerte el abandono dt estas residencias. De otro lado, el caló es prohibido, su uso podía llevar a galera: por varios años.

En 1783 se concreta la última ley de importancia sobre los gitanos, antes dt la época actual. Obra de Carlos III, es ya considerablemente liberadora, equipa. rándoseles prácticamente a los demás. La ley va a tener escasa vigencia ya que e rey muere en 1788 . Un año después, la Revolución Francesa atraerá hacia aque país a no pocos gitanos.

En el siglo XVIII se ordena realizar un censo gitano en España, que va c llevarse a cabo en dos fases durante los años 1784 y 1785 . Muy resumidamente el resultado más significativo indica que la población gitana española se concentra en un área de Andalucía sobre todo, la formada por las localidades de SevillaTriana, Morón, Marchena, Jerez, Arcos, Cádiz, Sanlúcar de Barrameda, Puertc Real y San Fernando. Son los núcleos en efecto más poblados, dándose la circunstancia de que casi ninguno de ellos es de asentamiento obligado.

Se sospecha que los gitanos, que estaban yá asentados en estos lugares andaluces desde bastante antes de que se ideara realizar el censo, habían sustituido a los moriscos en sus labores (artesanía por ejemplo). Ello no significa identificación acentuada entre ambos pueblos ya que son claras sus diferencias: hubo gitanos que participaron en lá represión contra los moriscos; los moriscos no consumen carne de cerdo y los gitanos tienen reparos para consumir carne de vaca, etc.

Pero lo cierto es que numerosos elementos gitanos se establecen en Andalucía y lo hacen legalmente no sólo como "castellanos nuevos" sino en ocasiones también como "castellanos de sangre vieja", argumentando para ello que descienden de caballeros que lucharon en Flandes para el Imperio.

Unos y otros se mezclaban con la población andaluza, una población que comenzó a llamar "flamencos" a los "elementos viejos" procedentes de Flandes o que, al menos, afirmaban proceder de aquellas tierras. $Y$ es en este contexto de finales del XVIII e inicios del XIX cuando va a nacer el cante flamenco.

Sostiene el profesor Leblon que en los siglos XVIII-XIX el 77 por ciento de los gitanos españoles reside en Andalucía, cultivan la tierra pero también los conocidos como "oficios prohibidos" (chalaneo, fragua...). Afirma Bemard Leblon que en Andalucía se refugian muchos gitanos huidos de sus obligadas residencias y recalca un dato esencial: gitanos hay en muchos lugares pero sólo 
en Andalucía se da el flamenco con intensidad, debido a un fenómeno especial de ósmosis. Un cante flamenco al que, repetimos, se le suponen raíces más o menos acertadas procedentes de la liturgia bizantina -poco conocida-, de la liturgia cristiana, de la música andalusí y de la húngara. La procedencia sobre todo a partir de esta última ha sido y continúa siendo investigada por profesores magiares sobre textos en húngaros y en cáló musicados, observándose unas pautas muy similares.

Al margen ya del pensamiento del profesor Leblon, casi toda la población española de raza gitana, que puede cifrarse en algo más de 300.000-400.000 personas vive actualmente en condiciones precarias. Se ha salvado una minoría por medio del flamenco, de pequeños comercios, anticuarios o representantes de marcas comerciales. A causa de los procesos socioeconómicos, parte de la población gitana tuvo que abandonar sus ocupaciones tradicionales como eran la forja, el chalaneo o la cestería. Persiguieron entonces algunos puestos eventuales de trabajo, siempre sin especialización y ganando a menudo sueldos inferiores a los payos. El porcentaje de gitanos analfabetos es asombroso; actualmente se da la cifra del 70-80 por ciento de la población e incluso más.

Por otra parte, el hecho de vivir en chabolas o en lugares de escasísimas condiciones asépticas trae consigo unas consecuencias dramáticas. Aunque es cierto que se dan esfuerzos por parte de los ayuntamientos para que esta situación al menos sea paliada, lo cierto es que la gravedad de la misma aún es patente. Félix Grande da unas cifras que no pueden pasarse por alto. Los procesos bronco-pulmonares afectan, durante el período invernal, al 90 por ciento de la población infantil; los procesos reumáticos al 85 por ciento de los mayores de 55 años. Y, en fin, solamente el 3 por ciento de la población total alcanza la edad de sesenta años o algo más ".

He aquí un panorama que trataremos de concretar más con el estudio de la localización de un asentamiento gitano en la misma Sevilla: el denominado de "Los Bermejales", verdadero apartado social ejemplo de marginación y no en exclusividad para gitanos.

\section{EL MINISTERIO DE ASUNTOS SOCIALES Y LA COMUNIDAD GITANA}

Los datos oficiales más recientes que hemos podido conseguir cuando escribimos estas líneas -primeros de junio de 1990- proceden del Ministerio de 
Asuntos Sociales y, en algunos aspectos, contrastan con algunos que se ofrece rán más adelante. La política básica, las líneas inspiradoras de la actuaciót administrativa central respecto a la etnia gitana puede decirse que no difieren $d t$ las de la Junta de Andalueía. Más bien se complementan sobre todo en $k$ financiero.

Ofrecemos a continuación un texto del propio Ministerio de Asuntos Socia. les ${ }^{10}$ sobre sus líneas de acción para con la comunidad gitana. Un cuadro anexc especifica que el Ministerio piensa dedicar más de 103 millones de pesetas : Andalucía dentro de un Plan Piloto para la Integración Social del Pueblo Gitano Los datos sobre la población gitana se refieren obviamente a toda España perc nos son de gran utilidad para la Comunidad Autónoma andaluza.

La actual política del Ministerio de Asuntos Sociales respecto a la comunidac gitana, la principal minoría étnica que existe en nuestro país, se concreta en ur plan de intervención inmediata, el Plan Nacional de Desarrollo Gitano, por medio de tres líneas de acción: programas de inserción social; programas de apoyc a las asociaciones gitanas y programas financiados con cargo al $0,5 \%$ de] I.R.P.F.

\subsection{Datos socioeconómicos de la población gitana}

Aunque los estudios realizados sobre el número de personas que integran la población gitana no se ponen de acuerdo sobre una cifra, se calcula que hay entre 300.000 y 350.000 personas gitanas en nuestro país.

Su distribución por zonas geográficas y por sexo es muy similar al resto de la población, lo que rompe supuestos tópicos de aislacionismo. Sin embargo, sus pautas culturales se conservan. La población gitana es considerablemente joven: el 70\% tiene edades inferiores a 25 años y sólo el $2 \%$ tiene más de 65 años.

La tasa de natalidad es casi cuatro veces superior, la de nupcialidad y la de mortalidad 1,4 veces superior, mientras que la esperanza de vida es de ocho o nueve años inferior, todo ello respecto al resto de la población.

La tasa de crecimiento demográfico es la más alta del mundo lo que hace prever que duplique su población actual en apenas veinte años.

La movilidad geográfica y nomadismo alcanza sólo al 5\% apareciendo una fuerte emigración interprovincial del pueblo a la ciudad y una más moderada emigración interprovincial. Viven mayoritariamente en familia con un promedio de miembros por familia de 5,72.

10. Plan Piloto para la Integración Social del Pueblo Gitano. 
En relación a la vivienda, el $80 \%$ de las familias gitanas ocupa viviendas no mayores de 50 metros cuadrados. De estas viviendas son chabolas alrededor del $21 \%$, el $8 \%$ prefabricadas, el $26 \%$ de protección oficial y el $43 \%$ son otro tipo de vivienda.

El $26 \%$ de la población gitana es activa frente a un $53 \%$ de población inactiva y un $21 \%$ de población dependiente de los activos; los obreros manuales representaban el $45 \%$, los temporeros el $20,3 \%$ y los chatarreros el $14,8 \%$.

La educación del niño gitano va incrementándose y en el curso 87-88 se estimaba que la escolarización era:

- el $50 \%$ de los niños de 4 y 5 años.

- el $80 \%$ de los niños de 6, 7, 8 y 9 años.

- del 70 al $60 \%$ de los comprendidos entre los 10 y 12 años.

- del 50 al $20 \%$ de los comprendidos entre los 13 y 15 años.

Los índices de analfabetismo se sitúan alrededor del $49 \%$.

La aprobación de la Constitución supuso un giro radical en el tratamiento desde las instituciones públicas del colectivo gitano. De una situación de persecución y presunción de delincuencia, recogida en la Ley de Peligrosidad Social, se pasó al reconocimiento de su ciudadanía, al declarar nuestra norma máxima la igualdad de todos los ciudadanos, sin diferencias por razón de raza.

\subsection{Plan de desarrollo gitano}

En 1989, el Ministerio ha puesto en marcha un plan de intervención inmediata para la comunidad gitana, dirigido a corregir la situación descrita, de acuerdo con los objetivos de integración social y de promoción del asociacionismo gitano.

Las acciones más relevantes realizadas a través de la Dirección General de Acción Social de este Ministerio han sido el desarrollo de, en primer lugar, programas de inserción social.

Durante 1989 se pusieron en marcha 66 proyectos pilotos de intervención integral para la asistencia, promoción e inserción social de comunidades gitanas marginadas, en colaboración con las comunidades autónomas. Para la selección de estos proyectos se han tenido en cuenta los criterios de participación de los propios afectados y de favorecer la convivencia entre payos y gitanos.

Los proyectos financiados han incluido: apoyo social y de monitores a gitanos para animación comunitaria; campañas de sensibilización y actividades culturales que favorezcan la convivencia payo-gitana. 
Esta colaboración con las comunidades autónomas se ha puesto en march con un presupuesto por parte de la Administración Central de 500 millones c pesetas para estos 66 proyectos que son cofinanciados por parte de las comun dades autónomas.

\subsection{Apoyo al asociacionismo}

El Plan contempla, en segundo lugar, programas de apoyo a las asociacionє gitanas.

Se han financiado programas y actividades realizados por entidades de ámb to estatal en favor de la comunidad gitana, cuyos objetivos concretos han side reforzar a las asociaciones gitanas a fin de implicar a los propios afectados en 1 lucha y promoción de derechos; potenciar el conocimiento e información d colectivo gitano en la sociedad mediante publicaciones, estudios e investigacic nes y formación de dirigentes gitanos.

En este capítulo se han invertido, durante 1989, 65 millones de pesetas distr buidos entre seis asociaciones gitanas de ámbito estatal: Enseñantes con Gitano: Secretariado Nacional Gitano, Integración Gitaná, Unión Romaní, Presencia G tana y el periódico quincenal de noticias del pueblo gitano "Nevipens Romaní" editado por la Unión Romaní en la que se federan gran parte de las asociacione gitanas.

\subsection{Programas con cargo al 0,5 del I.R.P.F.}

El año 1989 fue el primer año que se subvencionó a entidades gitanas co cargo a la asignación tributaria del impuesto sobre la renta por un total de 1 millones y medio de pesetas destinados a un proyecto de promoción integral d la comunidad gitana basado en un proceso educativo integral llevado a cabo pc la Federación Española de Universidades Populares.

Para 1990, en la convocatoria para la distribución de fondos provenientes d la declaración de la renta de 1989, el Ministerio ha incluido, dentro de la prioridades, a la minoría gitana y otras minorías con problemas sociales. 


\section{DATOS RELATIVOS A LA PRESENCIA DEL PUEBLO GITANO EN ANDALUCIA Y ACTUACION DE LA JUNTA}

Según un estudio de la Junta de Andalucía ${ }^{11}$, en la Comunidad Autónoma andaluza residen 250.000 gitanos aproximadamente. Este dato puede parecer exagerado por cuanto el "Libro Blanco de los gitanos españoles" 12 consigna para toda España la cifra de 210.000 gitanos con un margen de error del 10 por ciento más o menos. De manera que la cifra de la Junta de Andalucía estaría o resultaría excesivamente abultada ya que no creemos que todo el pueblo gitano esté habitando en Andalucía, sino que las bolsas de Barcelona y Madrid son también bastante importantes ${ }^{13}$.

Sea como fuere, la propia Junta afirma que los gitanos andaluces se concentran preferentemente en poblaciones mayores de 100.000 habitantes. La estructura de esta población se caracteriza por una media de edad de gran juventud (21,9 años), siendo así que las dos terceras partes de la población gitana andaluza es menor de 25 años. Raramente el gitano llega a ser anciano (en Andalucía sólo el 1,6 por ciento de la población gitana tiene más de 65-70 años). La media paya es del 10 por ciento.

La composición media de la familia gitana en Andalucía se sitúa muy cerca de los seis miembros, lo que supone unos 4 hijos por matrimonio, muy por encima de la media española paya que es de 2,3 hijos por matrimonio. Casi 7 de cada 100 familias gitanas tienen más de 10 miembros.

Las familias son relativamente estables en su residencia, pero cerca del 20 por ciento de ellas han vivido en tres o más puntos de residencia. Los ingresos familiares son muy bajos. La media se sitúa por debajo de las $30.000 \mathrm{pts} . / \mathrm{mes}$.

Hay un elevado índice de analfabetismo ( 73 por ciento), y un alto porcentaje de niños, en edad escolar, sin escolarizar (53 por ciento). Entre los escolarizados existe un gran porcentaje de absentismo y fracaso escolar. Los niños comprendidos en edad preescolar, están sin escolarizar en un cien por cien prácticamente.

En cuanto a la vivienda, aproximadamente la mitad (44 por ciento) de las familias gitanas viven en chabolas, casas en ruinas o en cuevas. La vivienda

11. Datos de 1987. Dirección General de Servicios Sociales. Plan Andaluz para la Comunidad Gitana. Incluye sondeo.

12. "Los gitanos españoles: Libro Blanco". Editado por el Secretariado Nacional Gitano, Torejón de Ardoz, Madrid, 1982.

13. Es importante consignar la dificultad existente a la hora de elaborar un censo gitano debido a la movilidad de esta etnia y a sus grados de nacimientos y defunciones. Se suele dar la cifra de entre 300.000 y 400.000 gitanos, para toda España según manifestaciones que hemos recogido en la Consejería de Fomento y Trabajo, y según datos oficiales del Ministerio de Asuntos Sociales. 
gitana, que sólo un tercio posee en propiedad, está situado en cascos viejc deteriorados, zonas de chabolas y barrios obreros, y es, mayoritariamente, d exiguas dimensiones.

Una quinta parte de las viviendas no tienen agua corriente, el 40 por ciento n posee cuarto de baño y carecen mayoritariamente de agua caliente, lavador: teléfono, TV y calefacción. La mayoría de los gitanos están insatisfechos con lugar donde viven, y se quejan de deficiencias en la infraestructura urbanístic del barrio y de los servicios de enseñanza y sanidad.

Por lo que al trabajo se refiere, es éste el principal problema de la comunida gitana. En el 64 por ciento de las familias no hay ningún trabajador fijo, y par un porcentaje similar de personas resulta el problema principal. Ente los parado: el 90 por ciento no recibe el subsidio de desempleo, si bien la situación podrí variar con el salario social. Entre los servicios que ellos creen que deben prestá seles con mayor urgencia, la búsqueda de trabajo resulta el principal.

Por último, en Sanidad, el 61 por ciento de las familias gitanas no tiene prestaciones ni cobertura médica de la Seguridad Social. El 17 por ciento de la familias tienen en su seno uno o más miembros minusválidos o enfermos crón] cos.

Según el citado "Libro Blanco", el 53 por ciento de los gitanos se encuentr ubicado en siete provincias: Granada, Barcelona, Madrid, Alicante, Sevilla, Jaé y Murcia. En la zona Sur vive el 42 por ciento de la población gitana española lo que representa un 1,2 por ciento en relación con la paya. Los valores porcer tuales de varones y mujeres son muy equilibrados $(51$ y 49 por ciento respectiv $\varepsilon$ mente). Las migraciones interprovinciales aparecen con índices máximos d desplazamientos en Barcelona y Madrid.

Hay que apuntar que la Administración Autónoma andaluza destina anual mente en sus Presupuestos entre 40.000 y 65.000 millones de pesetas orientada a servicios sociales, capítulo en el que se encuentran gitanos y payos pero e buena medida los primeros. Este presupuesto de servicios sociales se subdivid en programas sectoriales, programas comunitarios, pensiones, y subvenciones las corporaciones locales. Todos estos datos oficiales, hechos públicos en 1989 1990 por la Consejería de Hacienda, deben ser ahora tomados con alguna cautel debido a la ampliación del número de beneficiarios de la Seguridad Social y a denominado salario social al que ya se hizo referencia. ${ }^{\text {I4 }}$

14. Si tomamos como capítulo orientativo sobre el tema al presupuesto del Instituto Andaluz de Serv cios Sociales, tenemos que en 1989 dicho presupuesto fue de 47.675 millones de pesetas. En 1990, esi presupuesto asciende hasta 62.247 millones. Naturalmente, hay que tener en cuenta los presupuestos c otros organismos, como el Servicio Andaluz de Salud (S.A.S.) con un presupuesto de casi 365.00 millones de ptas. en 1990, y el Instituto Andaluz de la Mujer (poco más de 1.000 millones de ptas.). E 
Finalmente, concluyamos estos datos globales con una referencia a otros indicadores antropológicos facilitados por la Dirección General de Servicios Sociales de la Junta de Andalucía ${ }^{15}$. Según estos indicadores, los gitanos andaluces desean en su gran mayoría integrarse entre los payos aunque más del 40 por ciento creen que los payos no estiman, respetan ni comprenden a los gitanos.

El sondeo que ha efectuado la Junta para llegar a estas conclusiones conlleva un resultado que, en esencia, coincide con nuestra experiencia en "Los Bermejales". Dicho sondeo ha confirmado la hipótesis de la gran adhesión que existe entre los gitanos a los valores de su cultura y a los organizativos de su familia.

Así, se afirma mayoritariamente que la última palabra y autoridad ha de ser la del "tío"; que los ritos gitanos deben conservarse; que la mujer debe en todo caso obedecer y respetar a su marido, así como conservar su virginidad antes del matrimonio.

Casi desde el inicio de la legislatura socialista fue elaborado un Plan Andaluz para la Comunidad Gitana. Con el paso de los años desde su elaboración (198384), dicho Plan ha ido más o menos desarrollándose y coordinándose con la Administración Central, en la actualidad con el Ministerio de Asuntos Sociales, al que ya nos hemos referido.

El Plan indica ${ }^{16}$ que sus principios generales de actuación pretenden abordar los diferentes problemas del pueblo gitano en Andalucía "desde una perspectiva integral y globalizadora, superando una política centrada sólo en acciones aisladas y asistenciales".

Esta política integral se entiende, entre otros factores, a través de actuaciones sectoriales y participativas del propio pueblo gitano. Los programas de trabajo del Plan abarcan acciones comunitarias (barriadas, salud e higiene, educación, urbanismo y vivienda, etc.); acciones sectoriales (asociacionismo, convivencia payo-gitano, programa de lugares de acogida, jornadas, seminarios, publicaciones, etc.), entre otros aspectos.

Tarea diferente a la actual -y muy compleja- sería estudiar el efecto positivo o negativo del Plan. Evidentemente, los datos oficiales hablan de resultados positivos. Al final de este trabajo se adjuntan dos cuadros con datos oficiales relativos a la aplicación del Plan durante el período 1987-1988.

En el primero de ellos se advierte que el coste de los Programas Comunitarios ha sido en el período señalado de unos 5.000 millones de pesetas. En el segundo,

estas partidas se encierra de alguna manera el poco o mucho apoyo que la etnia gitana recibe de la Administración Andaluza. De otro lado, el salario social fue regulado legalmente en mayo de 1990 por el Gabinete de Rodríguez de la Borbolla y situado en 30.000 pts./mes.

15. Plan Andaluz para la Comunidad Gitana. Resumen, p. 6.

16. Id., p. 12 en adelante. 
hay una constatación de Programas Sectoriales 87-88 por un valor de unos 12 millones de pesetas. No obstante, hay que tener en cuenta que los programa comunitarios no están dirigidos sólo a gitanos sino a barriadas en las que convi ven payos y gitanos.

\section{PRINCIPALES CARACTERISTICAS DEL ASENTAMIENTO GITANO DE "LOS BERMEJALES" (SEVILLA)}

El asentamiento gitano de "Los Bermejales" se encuentra en el extrarradio di Sevilla, concretamente en el margen de una carretera perpendicular a la autovíi Sevilla-Cádiz, que conduce a la zona portuaria y al barrio de Los Remedios. Est último dato es importante puesto que los habitantes de "Los Bermejales" tienet en Los Remedios un foco de subsistencia biológica en base a los residuos margi nales del barrio -cartonaje sobre todo- y a otros factores, como la caridac religiosa de algunos de sus habitantes, que suelen apoyar perídicamente cor dinero o ropa las necesidades más elementales de los gitanos. La relativa proxi midad de Los Remedios también es importante por cuanto en unos terrenos in mediatos al barrio se asiente la Feria de Abril, acontecimiento que los habitante: de "Los Bermejales", sobre todo las mujeres, aprovechan para la mendicidad para vender flores o para "leer la buenaventura".

$\mathrm{El}$ asentamiento se compone de 26 familias que se corresponden con unć población de unos 125 habitantes, que viven en habitáculos prefabricados, artificiales, de material frágil y muy permeable a las variantes climatológicas. Es unc bolsa de indudable marginación estructural debido a una multiplicidad de causas factores étnicos, socioculturales, económicos, etc.

La población del asentamiento se distribuye de la siguiente forma:

- El 39 por ciento corresponde a población adulta (18-70 años), con características de analfabetismo, en paro, y cuya principal fuente de ingresos es la venta de chatarra, la mendicidad y ayudas de Cáritas.

- EI 5 por ciento corresponde a jóvenes (13 a 18 años), con iguales características que la población adulta, sin oferta de empleo, ni desempeño de oficio.

- EI 29 por ciento corresponde a niños de 6 a 13 años, no escolarizados y receptores de distintos niveles de deterioro.

- El 27 por ciento es población infantil de primera edad (0-6 años) receptores del marco de evolución y desarrollo que les posibilita su hábitat. 
Es común que, como mínimo, un matrimonio de "Los Bermejales" cuente con tres hijos, existiendo familias con ocho miembros ${ }^{17}$.

La población gitana que comentamos es oriunda de Sevilla. El asentamiento de "Los Bermejales" posee una antigüedad de 6 años. Anteriormente, sus habitantes estaban ubicados bajo el puente antiguo que conduce a la vecina localidad de San Juan de Aznalfarache. También se asentaron en chabolas frente a la factoría de Construcciones Aeronáuticas, S.A. (CASA), en Tablada, o en tiendas de campañas facilitadas por el Ejército.

Según testimonios de los habitantes de "Los Bermejales", la Policía Municipal procedió a la quema de sus chabolas hace unos seis años. Tras esta quema, sus habitantes fueron trasladados a su asentamiento actual, manifestándoles las autoridades municipales que tal asentamiento era provisional por el período de un año. Los gitanos se quejan pues de la tardanza en otorgarles una vivienda definitiva, así como de otros factores negativos que más adelante veremos.

Se da la circunstancia de que esta zona de Sevilla está destinada a unos determinados fines de expansión urbana según recoge el Plan General de Ordenación Urbana de Sevilla (PGOU). Por el momento, y en concreto, se ha presentado ya un proyecto para construir un centro hotelero en la zona. A pesar de todo, los responsables municipales aún no han manifestado claramente a los gitanos cuál va a ser su futuro destino, sembrando la consiguiente intranquilidad entre ellos. Al parecer, existen resistencias por parte de la población de determinadas barriadas periféricas de la ciudad para que los gitanos se asienten en ellas, a lo que se une el déficit de viviendas que Sevilla padece.

El Ayuntamiento de Sevilla ha dotado al asentamiento de una serie de servicios sociales a través de personal profesional, como un médico que visita periódicamente la zona, un psicólogo y un asistente social. La misión de estos profesionales es poner en pie un programa de educación tanto cultural como sanitaria, para lo que encuentran graves inconvenientes derivados de las necesidades económicas primarias de los gitanos así como de su factor consuetudinario y psicológico. Puede afirmarse que el resultado no ha sido positivo y que los profesionales encargados del asentamiento "se queman" en el intento de mejorar las condiciones de vida de sus habitantes.

A pesar de las manifestaciones contrarias al estado biológico-laboral de los gitanos, que hemos podido recoger y oír, la propaganda oficial afirma, entre otras teorías referentes al derecho de igualdad de oportunidades para todos, que "el resultado de la adaptación al nuevo entorno próximo se valora como positivo

17. Datos municipales inéditos facilitados a quien suscribe en el propio asentamiento por los profesionales contratados por el Ayuntamiento de Sevilla (1989). 
para todas las familias". Tal afirmación parece un sarcasmo teniendo en cuent que lo que personalmente hemos detectado es no un "acomodamiento" al luge sino una resignación y una adaptación forzosa al mismo.

Por lo demás, el asentamiento posee agua y luz que los gitanos no pagan causa de la "vista gorda" que realizan las empresas responsables del suministrc Por supuesto las calles carecen de asfalto o granito y se da la circunstancia d que existe en el lugar una colonia de perros que los gitanos utilizan para labore de caza, labor que llevan a cabo en los campos cercanos.

\section{DIVERSAS MANIFESTACIONES RECOGIDAS EN EL ASENTAMIENTO}

Aunque no hemos podido gozar del tiempo necesario para llevar a término u trabajo lo suficientemente profundo sobre el asentamiento gitano de "Los Ber mejales", sí podemos dar una idea sobre el aspecto mental y conceptual de su habitantes.

En primer lugar debemos destacar el bajísimo nivel cultural de los asentados hasta el punto de ignorar conceptos tales como "sexual", o "política". Ya hemo dicho que el nivel de alfabetización es muy bajo. No obstante, hemos podids saber que se ha dado una transmisión solidaria de conocimientos entre los pro pios gitanos. Así, quien tenía conocimiento de alguna regla gramatical lo hi transmitido a alguien de su familia o, simplemente, de su asentamiento.

Támbién es de destacar, aunque no sorprende demasiado, que estos habitante: de "Los Bermejales" no cuestionan para nada ciertos elementos o institucione: payas, salvo alguna excepción que veremos. Su adaptación a la mentalidad rei nante es considerable aunque tienen clara conciencia de su condición como raza diferente, que no inferior.

Tanto en los asentamientos que hemos citado antes -puente de San Juan d€ Aznalfarache, chabolas o tiendas de campañas- como en el actual, se da li presencia de ratas y otros animales perjudiciales por su peligrosidad sanitaria aspecto éste que los gitanos destacan en sus manifestaciones, unido al de la distancia respecto a Sevilla (Los Remedios) y, en la dirección a Cádiz, respectc al Hospital de Valme o, en el interior de la ciudad, el Hospital General, puntos en los que algunos elementos varones de "Los Bermejales" realizan tareas de guardacoches, que es otro de sus medios de subsistencia junto a la venta de cartones-chatarra y la limpieza de zapatos.

Estas labores son ejecutadas por los varones. Por su parte, las mujeres se desplazan a Sevilla a practicar la mendicidad y la venta de claveles. Asimismo. 
en el asentamiento cuidan de su vivienda, de los niños y de las labores propias del hogar. Es común, como es sabido, que las mujeres lleven consigo a algunos de sus hijos -normalmente al pequeño- para ejercer su tarea. En este terreno de lo laboral, una de las aspiraciones que poseen, sobre todo los hombres, es que les sea permitido sin tapujos por parte del Ayuntamiento el ejercicio de la venta ambulante.

Niegan los gitanos de "Los Bermejales" el tópico presente en algunos sectores sociales por el que se afirma que el gitano no desea desempeñar tareas laborales. Antes al contrario, insisten en la necesidad de ser apoyados por la colectividad para salir de su actual situación. No obstante, no desean trabajar a cualquier precio y bajo condiciones sublegales. Ante esta posibilidad, que se ha presentado en alguna ocasión, el gitano prefiere no depender de nadie y asegurar por sí mismo su supervivencia. Algunos de ellos, al hablar de puestos de trabajo, mencionan a lo que denominan "Partido de Andalucía", en referencia al Partido Andalucista (PA), para que les proporcione una ocupación digna.

Por otra parte, denuncian que determinadas tareas se entreguen a drogodependientes en lugar de a ellos, que hacen ostentación de no consumir droga. Igualmente, no son partidarios de que los delincuentes que abandonan la prisión gocen de seguro de desempleo porque, afirman, este hecho favorece el abstencionismo laboral y la delincuencia.

Reconocen que la situación socioeconómica es negativa para todos en general pero creen que ellos se ven especialmente castigados por esta situación, sosteniendo que nunca van a tener una "oportunidad" por su condición de gitanos.

Desconocían absolutamente cualquier episodio de la historia del pueblo gitano, aunque han podido acceder a ciertos aspectos de ella por medio de vídeos que los animadores sociales puestos por el Ayuntamiento les han proyectado. Gracias a estos vídeos afirman que ellos proceden de Egipto y de la India pero dicen, al mismo tiempo, que ellos no son los verdaderos gitanos sino que esa condición está reservada "a los húngaros" en referencia a los denominados zíngaros de Hungría. De todas formas, manifiestan que la tradicional represión que, contemporáneamente, ha ejercido sobre ellos la Guardia Civil, por ejemplo, en los momentos actuales se da en poca intensidad o no se da.

Coinciden en que aquellos gitanos que logran penetrar eri el tejido social de los payos y conseguir una consolidación socioeconómica en el mismo, se muestran insolidarios "con los que quedan abajo". Curiosamente, hablan de ellos como de "desgraciados", pero este calificativo no se pronuncia en el sentido de condición socioeconómica, sino ética, se pronuncia en un sentido de amoralidad para con sus compañeros de pueblo-raza. Al menos esto hemos deducido de nuestras conversaciones. 
Por lo que se refiere a la religión, hemos recogido testimonios que afirmar creer en las mismas cuestiones que los payos. Otros, por el contrario, matizabar este extremo: afirman creer en Dios pero no en los curas, a quienes raramente consideran solidarios de su situación. "Dios es Dios y un cura es una personi humana que está en este mundo", nos dijo un miembro varón del asentamiento Una mujer afirmó: "Para coger a un cura que nos ayude tenemos que buscarlc con un candil".

Se quejan, asimismo, de que no exista una iglesia en su asentamiento J denuncian el proceder de la congregación religiosa claretiana a la que acusan d haber hecho cuestaciones para apoyarles, habiéndose apropiado al final de lo re. caudado.

De la política afirman no entender, sin más. Algunos incluso ignoran qué e: la política y la propia democracia existente, sobre todo las mujeres. Admiten st indiferencia ante las ideologías, pretendiendo tan sólo que alguna dé fín a lś situación que padecen. En este sentido, creen que no tienen trabajo porque no Ic hay o porque lo hay "pero no nos lo dan". Constatan las diferencias de riquez: entre las clases sociales limitándose a expresar la insolidaridad "del rico hacia e pobre".

Son partidarios acérrimos del matrimonio -pasando a otro aspecto-, y recha. zan las relaciones sexuales fuera del mismo, así como el divorcio, separación y sobre todo, el aborto, todos estos temas impropios de la raza gitana, según hemos recogido. La fidelidad mutua es otro de los aspectos que, al menos en teoría consideran indiscutibles, al igual que, como se ha dicho, la indisolubilidad de matrimonio. "Una mujer debe ser para su marido hasta el morir", nos manifestc una gitana del asentamiento.

De otro lado, sostienen que la justicia es igual para todos, aunque no se refieren nunca a una justicia social -de la que no tienen clara conciencia sino tar sólo instinto de "estar mal"- sino, simplemente, a los tribunales de justicia, que dicen, deben castigar a quien comete un delito.

Respecto al Ejército, lo consideran necesario porque lo creen elemento defen. sor "de España". Reconocen al servicio militar como necesario. No hemos detec. tado conciencia antimilitarista en el sentido de criticar el estatus social del mili. tar y, mucho menos, en el de conocer organizaciones pacifistas o militar en ellas.

Se quejan de que, en ocasiones, los medios de comunicación exageran c tergiversan la realidad de la condición en la que se encuentran, colocándolos er un status de marginalidad y pobreza aún mayor que en el que están. "Han dichc por la televisión que aquí comemos en latas y mientras comemos nos estamos arrancando piojos. Eso es mentira, somos pobres pero no tanto", han coincidide varias manifestaciones. 
Por último, no existe en este asentamiento un liderazgo claro por parte de alguno de sus miembros. Lo que se da es la aptitud más decidida de unos miembros sobre otros a la hora de acometer acciones. Por otro lado, coinciden al afirmar que el cante flamenco es algo inseparable del gitano. "Lo llevamos en la sangre", nos han manifestado tres gitanas de diferentes edades. $\mathrm{Y}$ un gitano varón nos ha dicho: "Aprender a tocar la guitarra es más difícil, pero el cante no se aprende, lo llevamos dentro, sale de ti".

\section{CONCLUSIONES}

- El pueblo o etnia gitana continúa siendo, en su mayor parte, un grupo marginal dentro de la sociedad española y andaluza.

- Su marginación se deriva de un doble vector causal: la propia idiosincrasia del grupo y las características socio-económicas de la sociedad en la que está.

- Su situación de minoría marginal se ve acentuada por su dispersión y la poca unidad que existe en el grupo a nivel organizativo.

- Posee este grupo una conciencia de padecer injusticias pero no tiene una clara de consciencia de su "infelicidad".

- El nivel cultural es bajísimo, así como el nivel de bienestar material. Su índice de natalidad es elevado.

- El grupo suele ser permeable a la ideología y la mentalidad dominante.

- Se observan tímidas iniciativas de la Administración para paliar la situación en que se encuentran. 


\section{ANEXOS DE CUADROS COMPLEMENTARIOS}

\section{PROYECTO PILOTO PARA LA INTEGRACION SOCIAL DEL PUEBLO GITANO EN ANDALUCIA}

$$
\text { LOCALIDAD }
$$

Granada

Alınería, Córdoba, Málaga

Sevilla, Granada

La Cárolina, Santá Fé,

Pinos Puente, Fuentevaqueros,

Atarfe, Benalua, Guadix

Todas las de Andalucía

Todas las de Andalucía

15 Barrios o localidades de Andalucía

\section{DENOMINACION \\ DEL PROYECTO}

Programa de actividades de los Centros para la comunidad gitana

29.300 .000

Programa de apoyo a la escolarización infantil

55.885 .170

Programa de potenciación del asociacionismo. Encuentro jóvenes gitanos

Programa "Gitanos y comunidad" vídeo educativo, conferencias, folletos y murales.

Cursillo formativo de animación sociocultural para monitores gitanos.

Programa "Convivencia payo-gitano" 6.000 .000

\section{TOTAL}

En el cuadro n." 1, figuran los Programas Comunitarios realizados, con el coste de cada uno de ellos. 


\section{PROGRAMAS COMUNITARIOS REALIZADOS DEL PLAN ANDALUZ PARA LA CO- MUNIDAD GITANA EN 1987 Y 1988}

\begin{tabular}{lrc}
\hline Nombre del Programa & Coste en 1987 & Coste en 1988 \\
\hline 1. Gitanos y Comunidad & 5.850 .000 & 3.000 .000 \\
2. Plan Integral en & & \\
Barriadas: & - & - \\
2.1. Asistencia Sanitaria & - & - \\
2.2. Promoción de la Salud & - & 286.267 .000 \\
2.3. Drogodependencias. & 2.300 .000 & - \\
2.4. Saneamiento medioambiental & 9.821 .725 & 1.950 .000 \\
2.5. Alfabetización de adultos. & 1.848 .300 .000 & 2.138 .000 .000 \\
2.6. Escolarización infantil. & - & - \\
2.7. Erradicación Jel chabolismo & 12.540 .000 & 85.687 .000 \\
2.8. Infraestructura Urbana. & 38.539 .260 & 59.884 .950 \\
2.9. Ayuda Social. & - & 150.000 .000 \\
2.10. Andalucía joven (juventud) & - & - \\
2.11. Equipamiento social. & 2.203 .617 .985 & 2.447 .277 .700 \\
2.12. Formación profesional y fomento del empleo & - & - \\
2.13. Venta ambulante & - & - \\
2.14 Potenciación Cultural & & - \\
TOTAL & - & - \\
\hline
\end{tabular}


PROGRAMAS SECTORIALES REALIZADOS DEL PLAN ANDALUZ PARA LA COMUNIDAD GITANA EN 1987 Y 1988

\begin{tabular}{lrr}
\hline Nombre del Programa & Coste en 1987 & Coste en 1988 \\
\hline $\begin{array}{l}\text { 1. Estudio de necesidades sociales } \\
\text { de la Comunidad Gitaná }\end{array}$ & - & 1.000 .000 \\
$\begin{array}{l}\text { 2. Potenciación del Asociacionismo gitano } \\
\text { 3. Convivencia payo-gitano }\end{array}$ & 39.423 .000 & 45.470 .859 \\
4. Consejo Andaluz Gitano & 5.0000 .000 & 5.000 .000 \\
5. Centros para la Comunidad Gitana & 800.000 & 620.000 \\
6. Jornadas, seminarios, publicaciones & 17.200 .000 & - \\
TOTAL & & - \\
\hline
\end{tabular}

Fuente: Informe de la Secretaría de Estudios y Aplicaciones de la Comunidad gitana. Enero 1989. 


\section{BARRIOS ANDALUCES AFECTADOS MAS GRAVEMENTE POR LOS PROBLEMAS DE LA ETNIA GITANA}

1. Los Almendros

2. La Chanca.....

3. El Puche

4. Las Moreras

5. Las Palmeras

6. La Cartuja-La Paz

7. Almanjayar

8. Sacromonte

9. Guadix y Comarca

10. Santa Fé

11. Pinos Puente

12. Huéscar

13. Atarfe

14. Motril

15. Fuentevaqueros

16. Marismas de Odiel

17. Torrejón.

18. La Magdalena

19. Los Tejares (Linares)

20. Cobrerizas

21. Alcaudete.

22. Martos

23. La Palma-Palmilla

24. Los Asperones

25. Estación del Perro

26. Puente de los Morenos

27. Polígono Sur

28. Torreblanca la Nueva

29. El Vacie

30. San Diego

31. Chapina

32. San Pablo

33. Los Perdigones

34. Los Bermejales
Almería capital
Almería capital
Almería capital
Córdoba capital
Córdoba capital
Granada capital
Granada capital
Granada capital
Granada capital
Granada capital
Granada capital
Granada capital
Granada capital
Granada
Granada
Huelva capital
Huelva capital
Jaén capital
Jaén
Jaén
Jaén
Jaén
Málaga capital
Málaga capital
Málaga capital
Málaga capital
Sevilla capital
Sevilla capital
Sevilla capital
Sevilla capital
Sevilla capital
Sevilla capital
Sevilla capital
Sevilla capital 